\title{
Rechtliche und technische Rettungsanker für Privatheit und Datenschutz
}
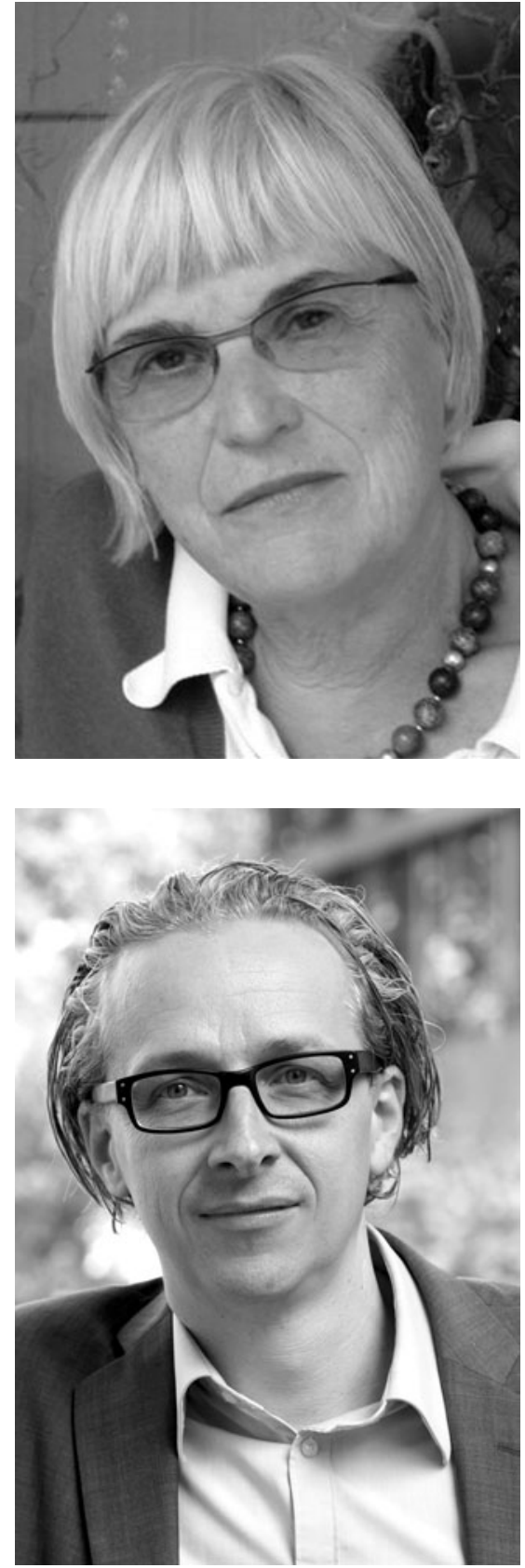

Das Urteil des EuGH vom 8. April 2014 zur Vorratsdatenspeicherung ist rechtspolitisch unmissverständlich und geht über die Ausführungen des BVerfG vom 2. März 2010 noch weit hinaus. Sabine Leutheusser-Schnarrenberger zeichnet die Vorgeschichte und das Ergebnis des bahnbrechenden Urteils nach, an dem sie als Menschenrechtlerin und ehemalige Bundesjustizministerin auf nationaler und europäischer Ebene nachhaltig beteiligt war. Das Urteil ist umso wichtiger, als insbesondere Geheimdienste Metadaten unbegrenzt sammeln und auswerten. Diese Art der Vorratsdatenspeicherung kann alles über das Leben des Einzelnen offenlegen. Welche hohen Risiken für die Privatsphäre damit einhergehen, bringen nicht zuletzt die Spitzenkräfte der NSA selbst mit ihren Äußerungen auf den Punkt: „If you have enough metadata, you don't really need content" (Stewart Baker 2014). „We kill people based on metadata" (Michael Hayden 2014).

Burkhard Schafer und Wiebke Abel befassen sich mit einer der überraschendsten Enthüllungen von Edward Snowden: die Überwachung populärer Online-Spielplattformen durch den englischen und amerikanischen Geheimdienst, die als „undercover Orcs" das Verhalten der Spieler psychologisch und kulturell beobachten und auswerten. Die Autoren zeigen, dass die öffentliche Debatte und Kritik an dieser Form der Überwachung im Vereinigten Königreich mehr vom Vorwurf der Verschwendung von Steuergeldern als von der Betroffenheit über die Verletzung der Privatheit und des Datenschutzes der Bürgerinnen und Bürger bestimmt ist. Damit wird einmal mehr die Notwendigkeit einer Verankerung der Privatheitsfrage in der europäischen Gesellschaft und einer EU-Grundverordnung deutlich.

Hans Joachim Hof befasst sich anwendungsbezogen mit der Frage nach den Grenzen des technischen Privatheitsschutzes. Er lässt keinen Zweifel daran, dass die Datenschutzdebatte, die sich teilweise auch in Kreisen von Datenschützern auf die Möglichkeiten von "sicheren" Verschlüsselungsverfahren konzentriert, das Problem allein nicht lösen kann, und dass datenschutzrechtliche Forderungen wie die nach Datenaskese weiterhin ihre Berechtigung haben. Seine detaillierten Darstellungen zeigen, dass die sog. "Allianz von Datenschutz und Technik" dringend einer praxisnahen Revision bedarf.

Mit Fragen der Datenminimierung und -anonymisierung befasst sich auch der Historiker und bisherige Leiter der Gedenkstätte für die Opfer der NS-Euthanasie-Verbrechen in Hadamar (Hessen), Georg Lilienthal. Dabei geht es um die sensible Frage, welche personalen Daten der Opfer nach datenschutz-, archiv- und strafrechtlichen Maßstäben in einem Gedenkbuch veröffentlicht werden dürfen, ohne die Rechte noch lebender naher Angehöriger zu verletzen, die öffentlich nicht mit einer erbbedingten Geisteskrankheit oder anderen "Stigmatisierungen“ in Verbindung gebracht werden wollen. Lilienthal berichtet aus Anlass der weiteren Debatte u.a. in Bayern auch von seinen Erfahrungen mit den Reaktionen von Angehörigen in der Gedenkstätte. Marie-Theres Tinnefeld nimmt diesen Faden für Bayern auf, wo, im Gegensatz zu Hadamar, Gedenkbücher öffentlich allen Bürger zugänglich gemacht werden. Die Gedenkbücher sollen den brutal Ermordeten ihren Namen und einen Ort des Ge- 
denkens geben, eine Forderung aus der Würde von Menschen, die als „unwertes Leben" vernichtet wurden.

In den USA existiert nur ein schwach ausgeprägter verfassungsrechtlicher Datenschutz, der sich primär gegen staatliche Stellen wendet. Anders ist die Situation in den einzelnen Bundesstaaten. Dort hat sich seit langem Kalifornien als "leader in information privacy" in den USA entwickelt. Matthias Orthwein und Katrin Anna Rücker erörtern am Beispiel von Data Breach, Do Not Track und dem Recht auf Vergessen, inwieweit die kalifornische Gesetzgebung sogar für Europa Vorbildcharakter haben kann. Das US-amerikanische Recht ist sodann auch Gegenstand des Beitrags von Simone Gräfin von Hardenberg. Ihr Thema ist die sog. Individualisierte Medizin in den USA, wobei im Mittelpunkt die Frage nach einem hinreichenden (Daten-) Schutz der dabei genutzten genetischen Gesundheitsinformationen steht. Zu Recht betont die Autorin, dass Patienten zur Preisgabe solch sensibler Informationen für Behandlungs-, aber auch für Forschungszwecke nur dann bereit sein werden, wenn durch klare rechtliche Vorgaben die informationelle Selbstbestimmung über diese Daten sichergestellt ist.

In der EU eskaliert derzeit der erbitterte Streit um das "Flaggschiff"-Projekt der Kommission, das Human Brain Project. Britta Schinzel setzt sich in ihrem Beitrag kritisch mit solcherlei „EU-Flagship-Projekten“ auseinander, die die europäische Grundlagenforschung in der Informations- und Kommunikationstechnik voranbringen sollen, dabei aber in gehörigem Umfang auf einer unreflektierten Big-Data-Philosophie beruhen.

Nicht in der großen weiten Welt, sondern in Bayern hat schließlich das Thema seinen Ursprung, dem sich Udo Kauß in seinem Beitrag zuwendet: die präventive Kontrolle des öffentlichen Raumes durch die automatische Kfz-Kennzeichenerfassung. Der Rechtsstreit um die bayerische Regelung ist aktuell beim Bundesverwaltungsgericht anhängig und es bleibt spannend abzuwarten, inwieweit die kritischen Anmerkungen des Autors dort auf offene Ohren stoßen werden.

\section{Marie-Theres Tinnefeld Benedikt Buchner}

